\title{
On Energy-, Angular Momentum-Loss and Pulsar Spark Gaps
}

\author{
A. Treves ${ }^{*, \dagger}$, M. Pilia ${ }^{*, \dagger}$ and M. Lopez Moya** \\ *Dipartimento di Fisica, Università dell'Insubria, Via Valleggio 11, I-22100 Como, Italy \\ ${ }^{\dagger}$ Affiliated to INAF and INFN \\ ${ }^{* *}$ Universidad Complutense, E28040 Madrid, Spain
}

\begin{abstract}
The neutron star spin down imposes a balance between the energy and angular momentum $(E / L)$ losses of a pulsar. This translates into constraints on the region of emission. The $E / L$ balance may be cogent in discriminating among the models of $\gamma$-ray production. Hence, models which require the release of the entire luminosity at the polar caps should be excluded. Also models where the radiative zone is well inside the speed of light radius have some difficulties. It is argued that a local unbalance of $E / L$ should generate a global instability of the magnetosphere, possibly quenching the emission at the polar caps.
\end{abstract}

Keywords: gamma-ray astronomical source - neutron stars - pulsars

PACS: $98.70 . \mathrm{Rz}, 97.60 . \mathrm{Jd}, 97.60 . \mathrm{Gb}$

\section{INTRODUCTION}

Since the seminal paper of Sturrock [1], the emission mechanism of pulsars is associated to spark gaps, regions where the parallel component of the electric field is so large that pair production may be copious.

A key issue treated frequently in the literature is the location of the gaps, with two favoured classes of models: a gap close to the star surface (polar cap gap), or a gap in the vicinity of the speed of light radius $r_{c}=c / \omega$ (outer gap). The advantage of the former possibility is that, close to the neutron star, the magnetic and electric fields are expected to be orders of magnitude larger than at $r_{c}$. On the other hand, as shown originally by Cohen $\&$ Treves and by Holloway [2,3] , photon release at the polar cap does not preserve the $E / L$ budget. This strongly favours models with the gap located at $r_{c}$ (e.g. [4]). However the double (or more complex) structure of pulsar pulses has generated new interest for models where both gaps are active (see e.g. [5, 6]). This is consistent with the observation that the various pulse components in general have different spectral shape $([7,8])$.

All previous considerations are essentially of little relevance for radio pulsars, since the fraction of the neutron star rotational energy released in the radio band is extremely small. The problem of the location of the emission regions becomes of renewed interest after the observation with the AGILE and Fermi satellites of tens of pulsars in the $\gamma$-ray band (see $[5,6,9])$, since in this band one observes a sizeable fraction of the spin-down energy (10-100\%, [9], see Figure 1). 


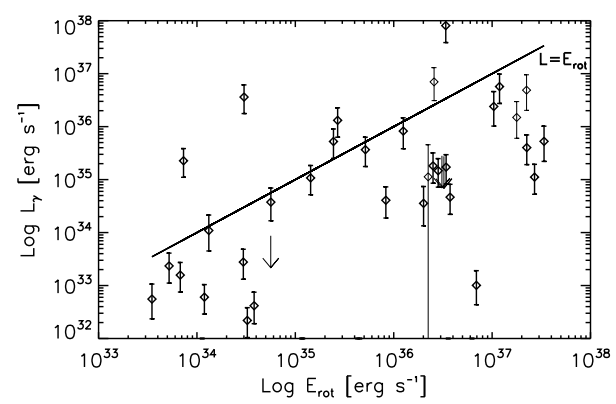

FIGURE 1. $\gamma$-ray luminosity of the pulsars observed by AGILE and Fermi as a function of the spin down energy loss (see also [9]).

\section{CONSEQUENCES OF THE ACTIVATION OF A POLAR CAP SPARK GAP}

Models could consider two spark gaps, one at the polar cap and one at the outer gap, and the $E / L$ balance could be globally preserved. This may occur also in the case of a continuous emission region (see e.g. the slot gaps [10]). For consistency, such models should clarify what is the physical mechanism which warrants that the luminosity distribution does preserve the $E / L$ balance.

Qualitatively one can make the following argument. If part of the energy is released at $r<r_{c}$, there is a local excess of angular momentum which one has to dispose of. The only solution we can think of is that the excess angular momentum is transferred to the magnetosphere, which expands to the periphery. The stretching of the magnetic field lines, may be a way of enhancing the radiation from an external gap, again favouring the global $E / L$ balance, possibly through a relativistic wind. However the appearence of a sizeable polar cap emission could rather induce some sort of global instability, which, one can argue, will quench the polar cap gap.

In conclusion we expect that the activation of a polar cap gap, if it ever occurs, should be followed by a) a modification of the relativistic wind, b) enhanced power from the outer gap, c) a global instability. The three effects may coexist or one or two may prevail. The alternative, which is simpler and we favour, is that there is no internal polar gap.

\section{REFERENCES}

1. P. A. Sturrock. Nature, 227:465-470, August 1970.

2. R. H. Cohen and A. Treves. A\&A, 20:305-+, August 1972.

3. N. J. Holloway. MNRAS, 181:9P-12P, October 1977.

4. K. S. Cheng, C. Ho, and M. Ruderman. ApJ, 300:500-539, January 1986.

5. A. Pellizzoni, et al. ApJ, 691:1618-1633, February 2009.

6. A. Pellizzoni, et al. ApJL, 695:L115-L119, April 2009.

7. A. A. Abdo, et al. ApJ, 713:154-165, April 2010.

8. M. Pilia, et al. ApJ, 723:707-712, November 2010.

9. A. A. Abdo, et al. ApJS, 187:460-494, April 2010.

10. A. G. Muslimov and A. K. Harding. ApJ, 606:1143-1153, May 2004. 